\title{
PVP Assisted Synthesis of Hydroxyapatite Nanorods with Tunable Aspect Ratio and Bioactivity
}

\author{
A. Joseph Nathanael, Young Ho Seo, and Tae Hwan Oh \\ Department of Nano, Medical and Polymer Materials, Yeungnam University, Gyeongsan 712749, Republic of Korea \\ Correspondence should be addressed to Tae Hwan Oh; taehwanoh@ynu.ac.kr
}

Received 30 November 2014; Revised 28 February 2015; Accepted 2 March 2015

Academic Editor: Subrata Kundu

Copyright ( 2015 A. Joseph Nathanael et al. This is an open access article distributed under the Creative Commons Attribution License, which permits unrestricted use, distribution, and reproduction in any medium, provided the original work is properly cited.

\begin{abstract}
Highly crystalline and reasonably uniform hydroxyapatite (HA) nanorods were prepared by polyvinylpyrrolidone (PVP) assisted hydrothermal synthesis which produces high aspect ratio (length/width) nanorods. The aspect ratio of the nanorods was higher in the presence of PVP and increased with increasing concentrations of PVP. X-ray diffraction (XRD) analysis showed that the HA nanorods were of the hexagonal apatite phase. High resolution transmission electron microscopy (HRTEM) and selected area electron diffraction (SAED) patterns confirmed that the nanorods grew in the $c$-axis direction (preferred orientation). The calculated lattice spacing was $\sim 0.35 \mathrm{~nm}$ which is the $c$-axis value of hexagonal HA. Morphological variations of pristine and PVP added HA were evaluated by field emission scanning electron microscopy (FESEM) and TEM, which revealed that the presence of PVP greatly increased the aspect ratio of the HA nanorods. The formation mechanism of the PVP assisted HA was studied and a possible reaction model was given. Cell viability analysis by in vitro studies showed encouraging results for the high aspect ratio nanorods and indicated a possibility for tuning the activity based on controlling the aspect ratio.
\end{abstract}

\section{Introduction}

The advancement of nanomaterial fabrication has led to the formation of high aspect ratio materials that have intrinsic, chemical, magnetic, and electrical properties enabling their interaction with biological molecules in a new way [1]. These one-dimensional (1D) nanomaterials (nanorods, nanowires, nanotubes, and nanobelts) have also led to great interests in scientific research owing to their efficient and enhanced basic properties. The most promising method for low cost production of nanoparticles with fine control over particle size, shape, and composition as desired is solution phase synthesis [2]. Many experimental parameters have to be optimized to obtain high aspect ratio nanoparticles. One approach to this is to add a capping agent to regulate the growth kinetics of the nanoparticles [3]. A major component of natural bone mineral is hydroxyapatite $\left(\mathrm{HA} ; \mathrm{Ca}_{10}\left(\mathrm{PO}_{4}\right)_{6}(\mathrm{OH})_{2}\right)$, which possesses good biocompatibility and osteoconductivity has been used in orthopedic and dental applications for decades [4]. The functional properties of the materials can be tuned by controlling their shape, dimensions, and size to attain the full benefits of that material in a specific field. Nano-HA has been shown to have higher specific surface areas than its bulk counterpart, which increases the adhesion of cells, protein, and drugs [5]. Some unique properties such as enhanced bioactivity, biocompatibility, and flexibility have also been reported for nanostructured-HA [6-8].

Polymer assisted nanoparticle synthesis has been reported for different materials in many studies. They are considered useful components for the preparation of one-, two-, and three-dimensional nanomaterials. The polymers used in the reaction change either the surface chemistry of the parent material or the concentration of soluble species that were already present to grow the crystal [9]. In our previous study, we reported the formation of hierarchically arranged flower like HA nanostructures by a hydrothermal method in the presence of polyacrylamide (PAM) [10]. In this study, we report the successful synthesis of high aspect ratio HA nanorods by hydrothermal method with the addition of polyvinylpyrrolidone (PVP). There have been some reports of PVP assisted synthesis of other nanoparticles such as $\mathrm{ZnO}$ and metal nanoparticles [11-13]. Additionally, 
TABLE 1: Different experimental parameters used to optimize the present work.

\begin{tabular}{lcclc}
\hline $\begin{array}{l}\text { PVP } \\
(\mathrm{g} / \mathrm{L})\end{array}$ & $\begin{array}{c}t \\
(\mathrm{~h})\end{array}$ & $\begin{array}{c}T \\
\left({ }^{\circ} \mathrm{C}\right)\end{array}$ & Morphology & Aspect ratio \\
\hline $\mathbf{0}$ & $\mathbf{1 2}$ & $\mathbf{1 8 0}$ & Uniform nanorods & $\mathbf{1 3} \pm \mathbf{3}$ \\
2 & 6 & 150 & Nanospheres & - \\
2 & 6 & 180 & Short nanorods & $7 \pm 3$ \\
2 & 12 & 150 & Nanorods and nanoparticles & $11 \pm 4$ \\
$\mathbf{2}$ & $\mathbf{1 2}$ & $\mathbf{1 8 0}$ & Nano- and microrods & $\mathbf{3 0} \pm \mathbf{8}$ \\
3 & 6 & 150 & Nanorods and nanoparticles & $8 \pm 2$ \\
3 & 6 & 180 & Short nanorods & $12 \pm 3$ \\
3 & 12 & 150 & Short nanorods & $15 \pm 4$ \\
$\mathbf{3}$ & $\mathbf{1 2}$ & $\mathbf{1 8 0}$ & Nonuniform & $\mathbf{4 3} \pm \mathbf{7}$ \\
4 & 6 & 150 & Nanorods & $25 \pm 4$ \\
4 & 6 & 180 & Nanorods & $28 \pm 3$ \\
$\mathbf{4}$ & $\mathbf{1 2}$ & $\mathbf{1 8 0}$ & Uniform nanorods & $\mathbf{5 6} \pm \mathbf{4}$ \\
4 & 12 & 200 & Thick and uniform & $45 \pm 2$ \\
4 & 24 & 180 & Thick and uniform & $43 \pm 3$ \\
\hline
\end{tabular}

a detailed analysis and the possible formation mechanism were discussed in detail. Finally, we investigated possible biomedical applications of this nanoparticle.

\section{Experimental Details}

2.1. Materials and Methods. Preparation of pristine HA nanorods was conducted as previously described [4]. Briefly, $\mathrm{Ca}\left(\mathrm{NO}_{3}\right)_{2} \cdot 4 \mathrm{H}_{2} \mathrm{O}$ and $\left(\mathrm{NH}_{4}\right)_{2} \mathrm{HPO}_{4}$ were separately dissolved in double distilled water. The $\mathrm{Ca}$ and $\mathrm{P}$ ratio were taken as the stoichiometric ratio of 1.67 of HA. The $\left(\mathrm{NH}_{4}\right)_{2} \mathrm{HPO}_{4}$ solution was then added to the $\mathrm{Ca}\left(\mathrm{NO}_{3}\right)_{2} \cdot 4 \mathrm{H}_{2} \mathrm{O} /$ polymer solution after which the $\mathrm{pH}$ of the solution was maintained at 9 by the addition of ammonium hydroxide (30\%). A milky white suspension was formed upon addition of $\left(\mathrm{NH}_{4}\right)_{2} \mathrm{HPO}_{4}$ solution. After adding all of the phosphate solution, the mixture was stirred vigorously for a few minutes and the obtained milky white suspension was transferred into a $50 \mathrm{~mL}$ Teflon-lined stainless steel autoclave and heated at $180^{\circ} \mathrm{C}$ for $12 \mathrm{hrs}$. The sample was then cooled to room temperature, after which the precipitate was washed several times with ethanol and distilled water. Finally, the powder was dried at $100^{\circ} \mathrm{C}$ and calcined at $400^{\circ} \mathrm{C}$ before further characterization. For PVP assisted synthesis, different amounts $(2,3$, and $4 \mathrm{~g} / \mathrm{L})$ of polymer (polyvinylpyrrolidone (PVP), MW15,000) were added to the calcium precursor solution. To identify the optimized conditions, the hydrothermal treatment time and temperature were changed (Table 1).

2.2. Characterization. The HA nanorods prepared by the polymer assisted hydrothermal method were analyzed by several methods. Structural analysis was conducted using X-ray diffraction analysis (XRD, Rigaku, D/MAX 2500H), while field emission scanning electron microscopic analysis (FESEM, Helios 600) was employed to identify morphological changes in response to the addition of PVP. Morphological and structural confirmation were conducted by transmission electron microscopy (FR-TEM, Tecnai F30 S-Twin) and selected area electron diffraction (SAED) analysis, respectively. Thermogravimetric and differential thermal analysis (TG/DTA) was conducted using a thermal analyzer (SDT Q600 V20, TA instruments) at a heating rate of $10^{\circ} \mathrm{C} / \mathrm{min}$ from room temperature to $800^{\circ} \mathrm{C}$ in a nitrogen atmosphere. FTIR analysis was performed using a FT-IR spectrometer (PerkinElmer Spectrum 100).

Human osteosarcoma cells (MG-63 cells) were cultured to determine the cell viability of PVP assisted high aspect ratio nanorods as described in our previous report [10]. Briefly, cells were cultured at $37^{\circ} \mathrm{C}$ in a humidified atmosphere of $5 \% \mathrm{CO}_{2}$ in air containing cell culture medium (DMEM media (phenol red free), Welgene), $10 \%$ fetal bovine serum (FBS, Welgene), $2 \mathrm{mM}$ L-glutamine, $1 \%$ penicillin, and streptomycin mixture (Antibiotic, Gibco). The cells were then seeded in 96-well microassay plates at a concentration of $1 \times 10^{4}$ cells/well and cultured for $24 \mathrm{~h}$. Sterilized HA nanoparticle samples were subsequently added to the wells at a concentration of $100 \mu \mathrm{g} / \mathrm{mL}^{-1}$ and cocultured for 1,3 , and 5 days. Finally, the cell proliferation or viability of cells was determined using a CCK-8 kit (Dojindo, CK-04-13). Five samples were tested for each culture time period and the mean values were reported.

\section{Results and Discussion}

3.1. X-Ray Diffraction Analysis. The XRD patterns of the pristine and PVP assisted HA nanorods are shown in Figure 1. Figure 1(a) shows the XRD pattern of pure PVP for comparison. Structural analysis revealed that all samples showed good crystallinity. Upon careful analysis, we found a small decrease in the intensity of the peak of PVP added samples (Figures 1(b)-1(d)) compared to pristine HA (Figure 1(e)). While increasing the polymer concentration the peak intensity was slightly increased. Conversely, the (002) diffraction peak of the hexagonal HA gained strong intensity, which may have been due to the preferential growth along the $c$ axis (001). Morphological analysis revealed that the growth along the $c$-axis direction was indeed clear. Hence, the PVP addition increases the preferential growth along the (002) direction which is the reason for the increase in the (002) diffraction peak observed upon XRD analysis. Apart from that, no additional impurity peaks were found due to the addition of PVP. All peaks were readily indexed with the HA hexagonal phase (space group: P63/m (176)). The calculated lattice parameter for $a$ was 9.417 while that for $c$ was 6.886 (JCPDS\#09-0432) [4]. There were no significant changes in the lattice parameters during polymer assisted synthesis.

The average crystallite sizes, $L$, of the samples were calculated using the Debye Scherer formula [14]:

$$
L=\frac{k \lambda}{\beta \cos \theta},
$$

where $k=0.94$ (shape factor), $\lambda$ is the wavelength of the radiation used (1.5405 $\AA$ ), $\beta$ is the full width at half maximum of the peak in radians, and $\theta$ is the Bragg angle. Highly intense 


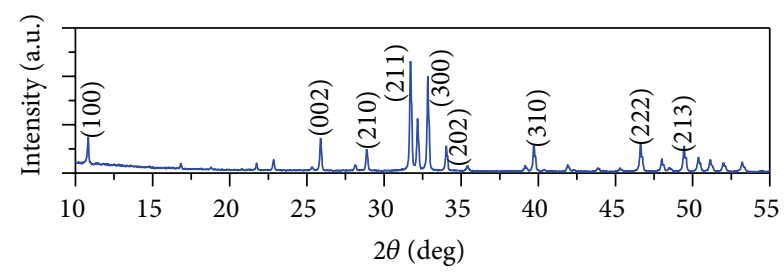

(e)

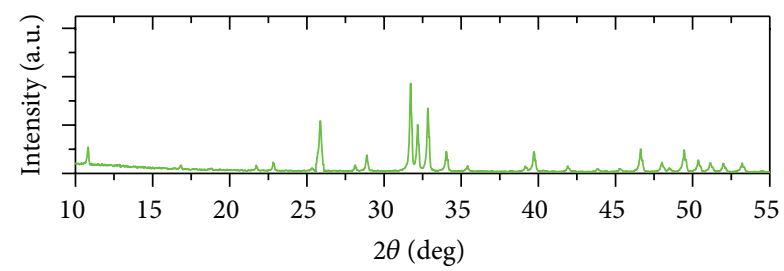

(d)

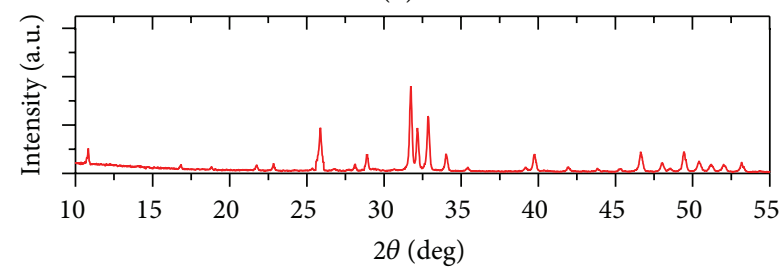

(c)

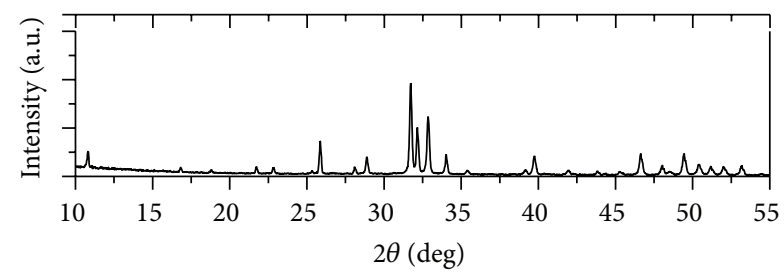

(b)

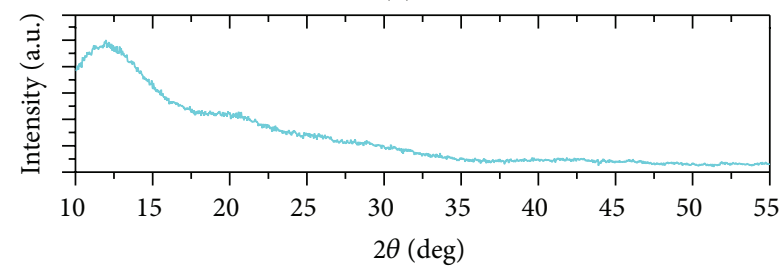

(a)

FIGURE 1: XRD pattern of high aspect ratio HA nanorods: (a) pure PVP, (b) 2, (c) 3, and (d) 4 g/L PVP addition, and (e) pristine HA.

peaks in the XRD patterns were taken into account for the calculation (Figure 1). The crystallite size increased from $17 \pm$ 5 to $37 \pm 4 \mathrm{~nm}$ as the polymer concentration increased.

The presence of PVP was not reflected in any HA samples. In a previous study of PVP added nanoparticles, such as $\mathrm{CdS}$ and $\mathrm{ZnO}$, even though the weight percent of PVP was higher than in the present study, no PVP peaks were observed upon XRD $[9,15]$. The same trend was observed in the present study. This may have occurred because the addition of PVP was followed by heat treatments and calcination at $400^{\circ} \mathrm{C}$ for $2 \mathrm{hrs}$. Hence, the PVP may have been removed at the time of calcination. To confirm the decomposition of PVP at the calcination temperature, we conducted TGDTA analysis for pure PVP and PVP added HA $(4 \mathrm{~g} / \mathrm{L}$
PVP added sample) (Figure 2). Under the same experimental conditions, pure PVP and PVP added HA samples showed very different trends. For pure PVP, the sample started to decompose at $380^{\circ} \mathrm{C}$, whereas decomposition started at $340^{\circ} \mathrm{C}$ for PVP added HA nanoparticles. Similar decrease in the decomposition temperature of PVP has been observed in previous cases of PVP added nanoparticles [16]. The initial weight loss for PVP was around $11.7 \%$ at $T_{\text {room }} \leq T \leq 80^{\circ} \mathrm{C}$ which may have been due to the loss of absorbed or hydration water molecules. The next complete weight loss of around $86.9 \%$ was observed at $200^{\circ} \mathrm{C} \leq T \leq 480^{\circ} \mathrm{C}$ due to the decomposition of PVP. For PVP added HA, a weight loss of around $3.8 \%$ was observed around $290^{\circ} \mathrm{C} \leq T \leq 430^{\circ} \mathrm{C}$ due to the complete decomposition of PVP at this temperature. These findings coincide with the reported data for PVP added nanoparticles.

FTIR analysis (Figure 3) of the samples revealed that the PVP added samples contained functional groups of HA rather than PVP at all stages. The characteristic bands for $\mathrm{PO}_{4}{ }^{3-}$ appeared at $472,583,601,961,1032$, and $1108 \mathrm{~cm}^{-1}$ for pristine and PVP added HA samples, while no other PVP functional groups were found in the PVP added HA sample. The FTIR analysis of pure PVP was shown in Figure 3(c). A peak at $1645 \mathrm{~cm}^{-1}$ is assigned to the stretching vibration of the $\mathrm{C}=\mathrm{O}$ [17]. $\mathrm{C}-\mathrm{H}$ bond in $\mathrm{PVP}$ is present at $1374 \mathrm{~cm}^{-1}$. Peaks at $1287 \mathrm{~cm}^{-1}, 1437 \mathrm{~cm}^{-1}$, and $1424 \mathrm{~cm}^{-1}$ are due to the $\mathrm{C}-\mathrm{N}$ stretching vibrations and the attachment of $\mathrm{CH}_{2}$ groups in the pyrrole ring of PVP [17]. The FTIR analysis clearly indicated that the small amount of PVP added during synthesis was removed during the calcination process and hence not detectable upon XRD analysis.

3.2. Field Emission Scanning Electron Microscopy. FESEM analysis was conducted on all samples to investigate the effects of PVP on HA morphology during the hydrothermal synthesis. Figure 4 shows the morphological variations in the HA nanorods. Even though the results of structural analysis did not differ greatly among samples, there were vast changes in the size of the nanorods. The pristine HA nanorods possessed an average length of $300 \pm 10 \mathrm{~nm}$ and a width of $22 \pm 3 \mathrm{~nm}$ (Figure 4(a)), whereas the sample prepared in the presence of PVP showed significantly greater lengths and widths. When compared to the width, the length of the nanorods was greatly increased, which increased their aspect ratios. Further increasing the PVP concentration resulted in additional increase of length, whereas the width decreased.

The length of the $2 \mathrm{~g} / \mathrm{L}$ PVP added sample was $2 \pm 0.3 \mu \mathrm{m}$ and the width was $60 \pm 10 \mathrm{~nm}$ (Figure 4(b)); hence, the aspect ratio of this sample increased to the mean value of 30 relative to the pristine sample value of 13 . For the sample prepared using $3 \mathrm{~g} / \mathrm{L}$ of PVP, the aspect ratio was further increased to the mean value of 43 because the length of the rod increased further to $2.4 \pm 0.3 \mu \mathrm{m}$ and the width decreased to $50 \pm 8 \mathrm{~nm}$ (Figure 4(c)). Further increasing the PVP concentration to $4 \mathrm{~g} / \mathrm{L}$ resulted in an increase of length up to $2.6 \pm 6 \mu \mathrm{m}$ and a reduction in width to $46 \pm 4$ (Figure $4(\mathrm{~d})$ ), which caused the aspect ratio to increase rapidly with respect to pristine HA. 


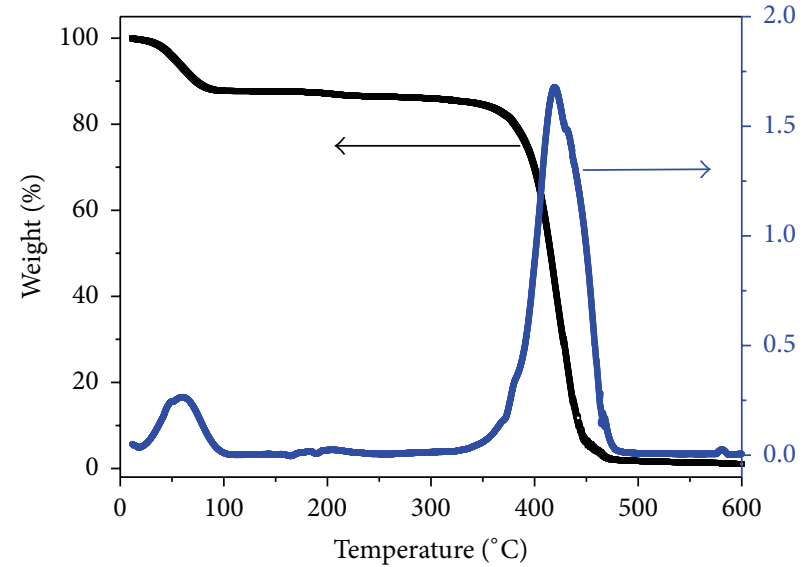

(a)

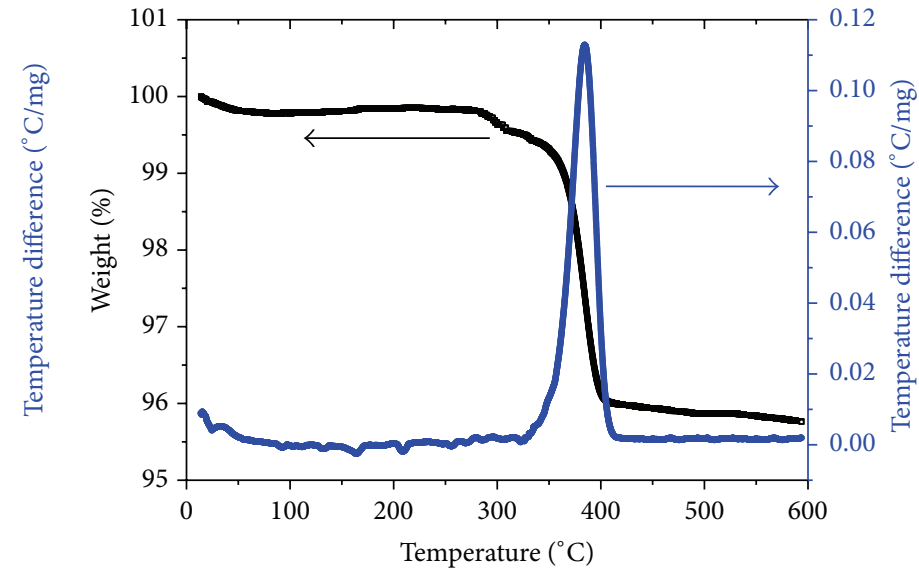

(b)

FIgUre 2: TG-DTA analysis of (a) pure PVP and (b) 4 g/L PVP added HA.

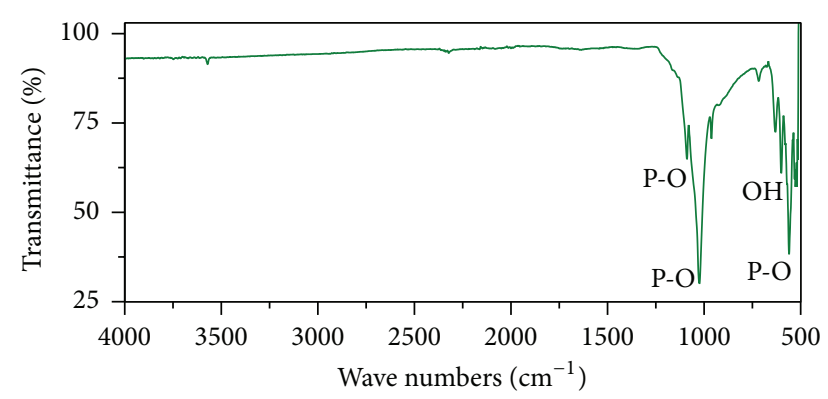

(c)

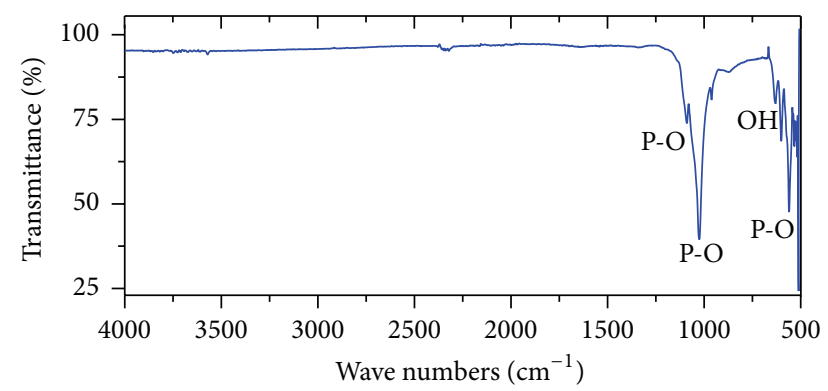

(b)

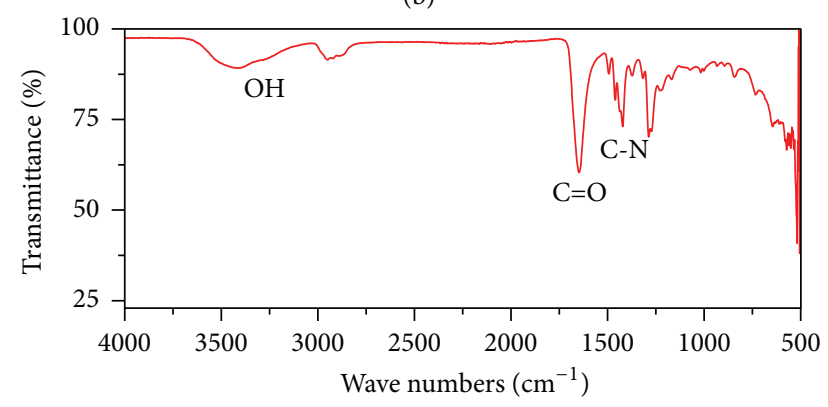

(a)

Figure 3: FTIR spectra of (a) pure PVP, (b) 4 g/L PVP added HA, and (c) pristine HA.
After the addition of PVP and increase in PVP concentration, growth along the $c$-axis direction was induced and the length of the nanorods increased. The $c$-axis directional growth was confirmed by the increase in intensity of the (002) peak in the XRD pattern (Figure 1). EDS analysis (Figure $4(\mathrm{e})$ ) of PVP added HA ( $4 \mathrm{~g} / \mathrm{L})$ confirmed the presence of $\mathrm{Ca}, \mathrm{P}$, and $\mathrm{O}$ by showing characteristic peaks of pristine HA. Other elements were not detected upon EDS analysis.

\subsection{Transmission Electron Microscope Analysis. Typical TEM} images are shown in Figure 5. The results confirmed that all samples had nanorod structures, indicating that the addition of PVP does not change the morphology. Pristine HA nanorods (Figure 5(a)) have shown some aggregation and an aspect ratio limited to around 13. In our previous study, we synthesized HA nanorods via a hydrothermal method by changing various parameters. However, we could not achieve a high enough aspect ratio, to produce an elongated structure that could interact with cells and biomolecules in different ways [1] to enhance their activity or enable tuning by controlling the aspect ratio. In the present study, TEM analysis revealed that adding PVP in a controlled manner can achieve a desired aspect ratio, thereby tuning the functional properties. For the $2 \mathrm{~g} / \mathrm{L}$ PVP added sample, the uniformity could not be obtained and there was a large difference in width (thickness) among nanorods (Figure 5(b)). Specifically, some nanorods had thicknesses of more than $200 \mathrm{~nm}$, and most had widths of around $120-130 \mathrm{~nm}$. Samples containing $3 \mathrm{~g} / \mathrm{L}$ added PVP showed increased uniformity and very few nanorods with different thickness. Overall the thickness of the nanorods in these samples decreased to around 80$100 \mathrm{~nm}$ (Figure 3(c)). Further increasing the PVP content to $4 \mathrm{~g} / \mathrm{L}$ resulted in increased uniformity of the width of the nanorods and a high aspect ratio of HA nanorods. The thickness of the nanorods was $50 \pm 10 \mathrm{~nm}$ and their lengths 


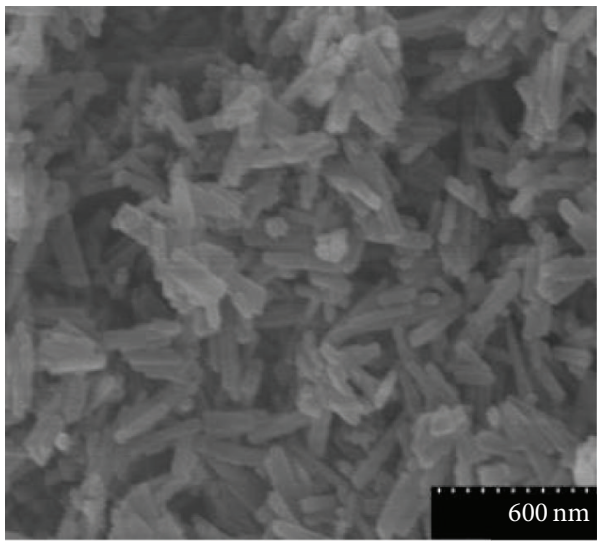

(a)

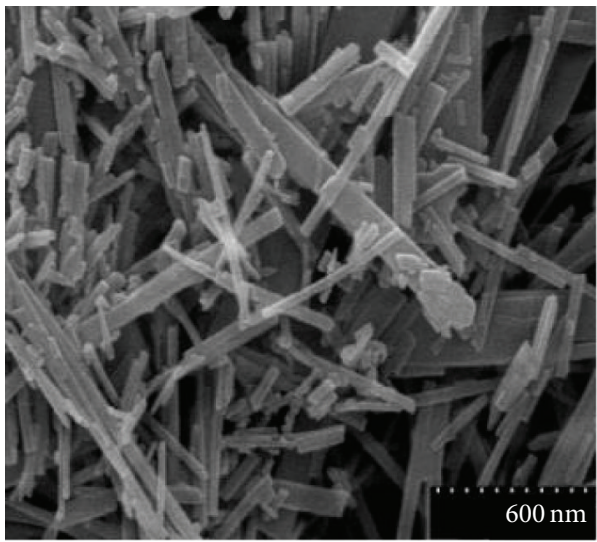

(c)

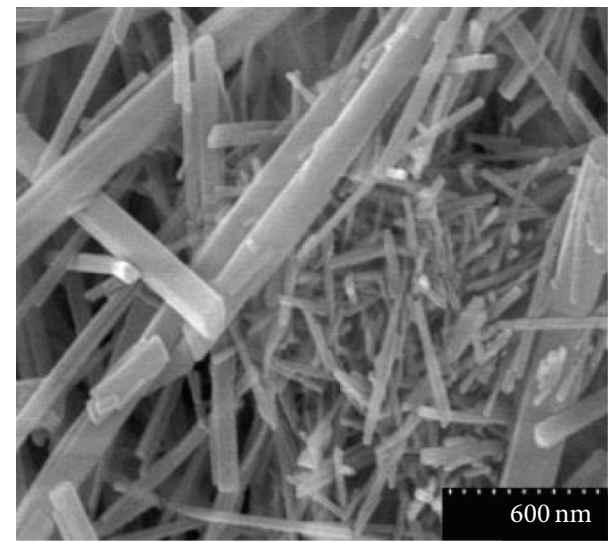

(b)

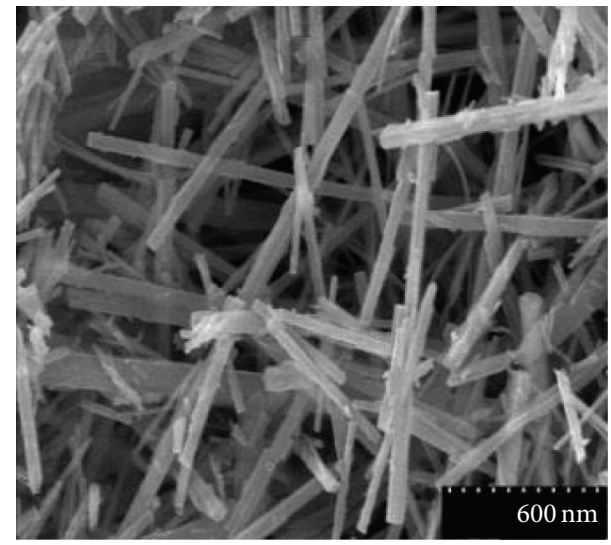

(d)

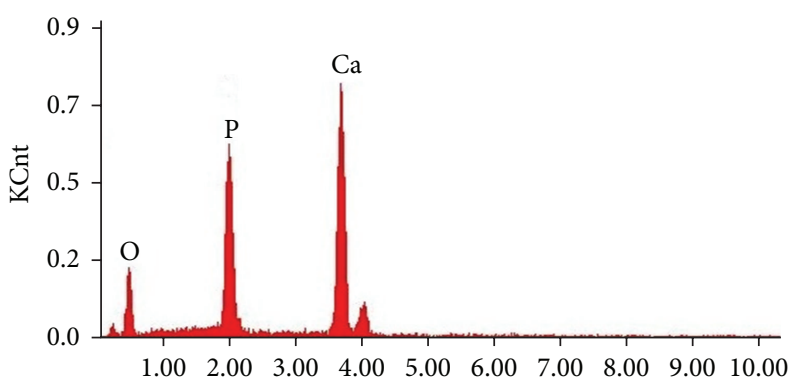

(e)

FIGURE 4: FESEM image of the high aspect ratio HA nanorods: (a) pristine, (b) 2, (c) 3, and (d) 4 g/L PVP addition, and (e) EDX pattern of (d).

were $>1 \mu \mathrm{m}$ (Figure 5(d)). Further increasing the PVP ratio destroyed the uniformity as well as the morphology of the product. As shown in Figure 5(e), HRTEM revealed a $d$ spacing value of around $0.35 \mathrm{~nm}$, which is the value of the (001) face of apatite. These findings indicate that the nanorods were grown in the $c$-axis orientation and confirmed the results of XRD analysis. Similarly, the SAED pattern of the nanorods revealed that the high aspect ratio nanorods possess a single crystalline nature as indicated by the clear diffraction spots (Figure 5(f)).
3.4. Possible Formation Mechanism. By carefully analyzing the results, we derived a possible mechanism for formation of the high aspect ratio of HA nanorods. PVP assisted nanoparticle synthesis has been described for many different materials, including $\mathrm{CdS}, \mathrm{ZnO}$, and metal nanoparticles [9, 11-13]. For $\mathrm{ZnO}$ nanorods, the amount and molecular weight of PVP have been reported to influence the morphology and growth of the nanoparticles. The aspect ratio also changed while changing the concentration of PVP. It has been reported that the amount of PVP in the reaction assisted the 


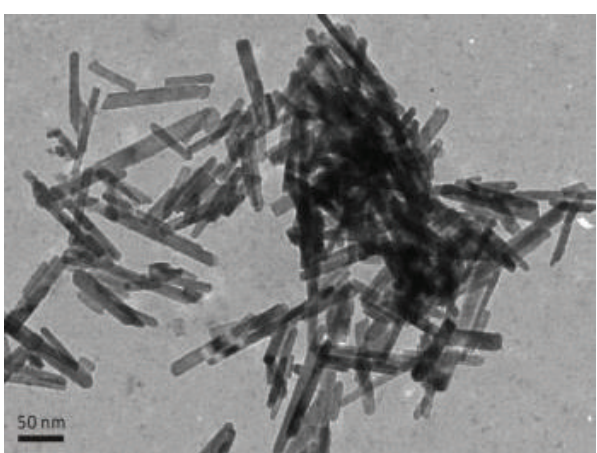

(a)

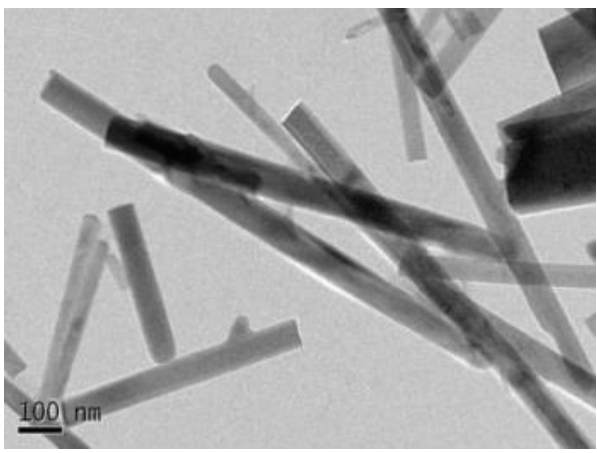

(c)

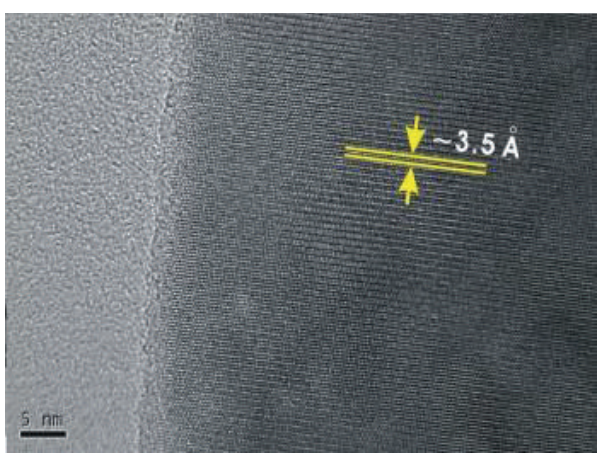

(e)

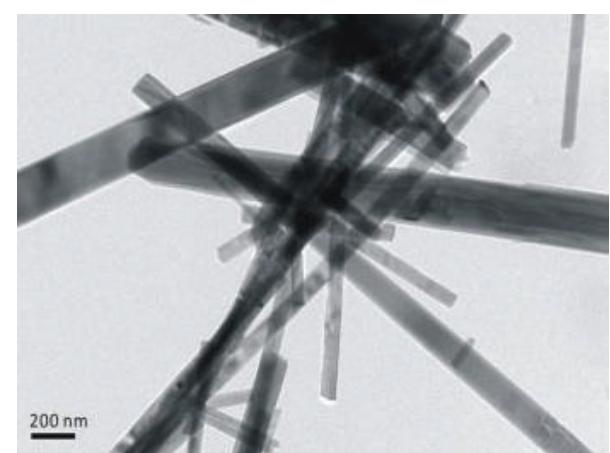

(b)

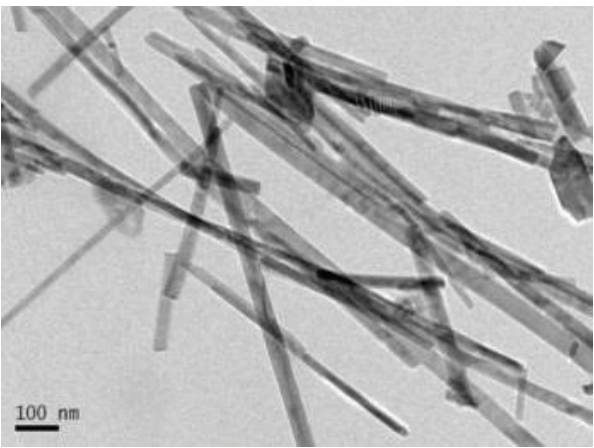

(d)

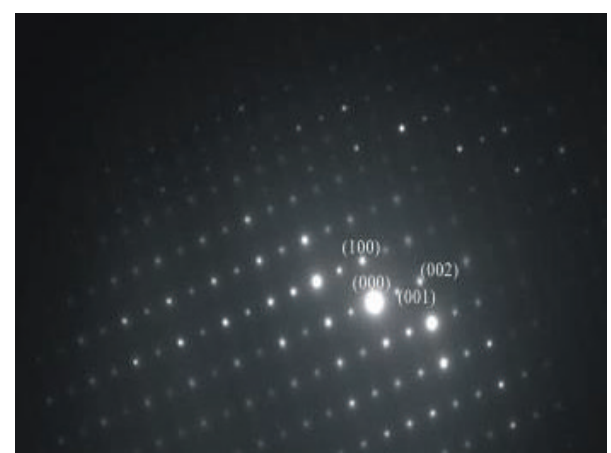

(f)

Figure 5: TEM image of the high aspect ratio HA nanorods: (a) pristine, (b) 2, (c) 3, and (d) 4 g/L PVP addition, and (e) and (f) HRTEM and SAED pattern of $(\mathrm{d})$.

controllable nucleation and growth of $\mathrm{ZnO}$ which makes it possible to control the size. In this study, we attempted to control and tune the size and uniformity of the product by changing the PVP concentration in the reaction mixture. In the HA/PVP system, it is assumed that the $\mathrm{C}=\mathrm{O}$ of the PVP molecule might have been absorbed to $\mathrm{Ca}^{2+}$, after which the growth was controlled by the PVP. For the CdS nanorods, PVP has been reported to have stronger interactions with the side faces than the ends, which influences growth along the $c$-axis direction [9]. This was also true in the present study, with increasing the PVP concentration resulting in greatly reduced thickness and increased growth along the $c$ axis. Therefore, these findings suggest that the addition of PVP restricts growth along the side faces by capping them heavily while activating growth along the end direction (caxis), resulting in a high aspect ratio growth of HA nanorods. The preferred orientation growth along $c$-axis and $a$-axis is shown in Figures 6(a) and 6(b). For a lower amount of PVP samples, we found that the uniformity in the thickness of the nanorods was restricted. This may have been due to the limited availability of the PVP molecules to cap the side faces. When increasing the PVP, a uniform distribution of nanorods was obtained; however, further increasing the PVP content destroyed the morphology and uniformity. These findings clearly show that PVP played a major role in controlling the growth direction and uniformity. A schematic illustration of the formation mechanism in the presence of PVP is shown in Figure 6(c). Hence the growth in the side surface was 


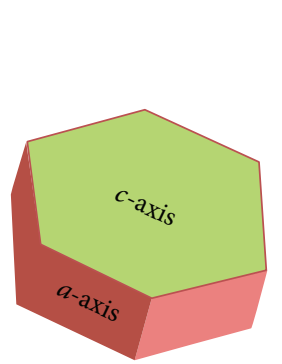

(a)

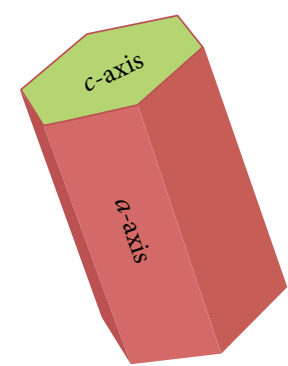

(b)

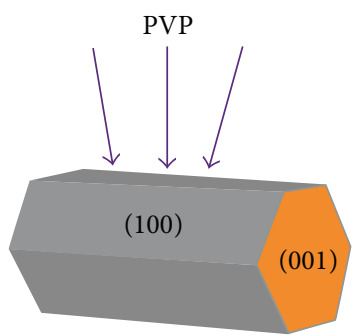

(c)

FIGURE 6: HA nanoparticle preferred orientation to (a) $a$-axis, (b) $c$-axis, and (c) possible high aspect ratio nanorod formation in the presence of PVP. The end faces of the nanorods were in a (001) orientation, while the side faces were in a (100) orientation. The strong interaction between the PVP on the (100) face is shown in gray color and the weak interaction on the (001) face is shown in orange.

completely suppressed by the PVP, while growth was induced at the two ends of the nanorods.

It has also been reported that the presence of PVP prevents the aggregation of nanoparticles [18], which was clearly observed in the present study. The pristine HA nanoparticles aggregate heavily, whereas the PVP assisted HA nanoparticles showed very little aggregation and many separate nanorods. Moreover, the addition of PVP kinetically controlled the growth rates of different faces of the nanoparticles and induced the growth of longer nanorods. We are attempting to obtain a clearer picture of the growth mechanism through further work and analyses.

3.5. In Vitro Cellular Analysis. Nanometer scale materials are particularly interesting in biology since the dimensions of many important subcellular structures fall in the 1-1000 nm range. Several research groups have investigated use of high aspect ratio nanoparticles for biological applications such as protein detection, glucose sensing, and gene delivery [1]. Good transfection efficiency and potential application have been reported for genetic vaccination applications. The present study was designed to use these high aspect ratio nanorods for implant applications. In this study we conducted in vitro cellular analysis using MG-63 cell lines to compare the efficiency of these high aspect ratio nanorods for possible implant application. Figures 7 and 8 show the respective cell viability analysis and morphology of MG-63 cell lines for different nanoparticles. Pristine HA nanorods showed good cell viability for different time periods. However, when the nanoparticles were prepared by the addition of $2 \mathrm{~g} / \mathrm{L}$

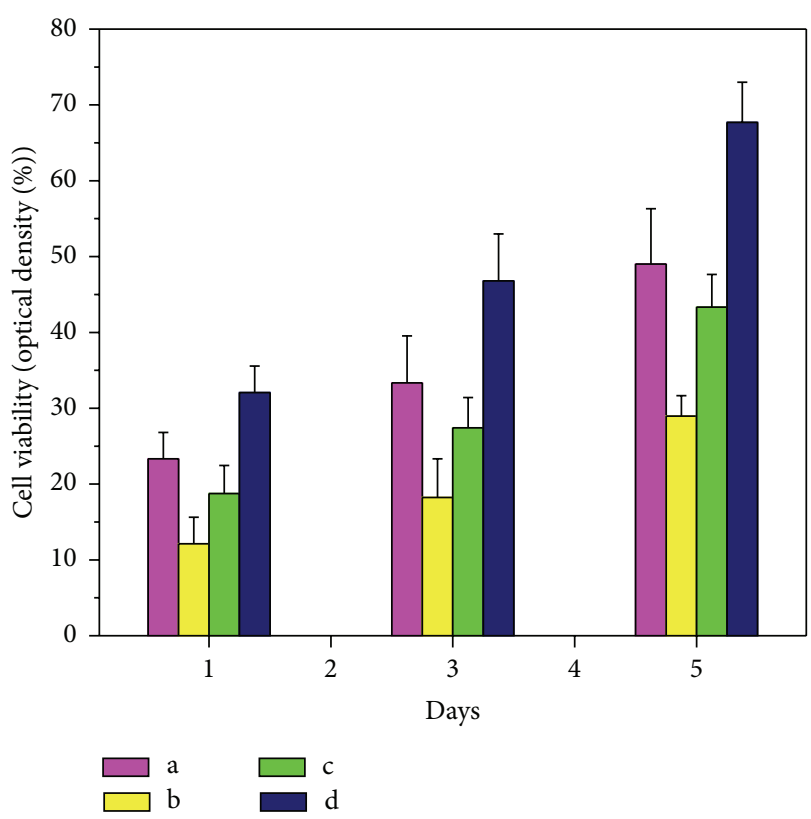

FIGURE 7: MG-63 cell viability on the high aspect ratio HA nanorods: (a) pristine and (b) 2, (c) 3, and (d) 4 g/L PVP addition.

PVP, the viability decreased suddenly. Further increasing the PVP content to $3 \mathrm{~g} / \mathrm{L}$ PVP gradually increased the cell viability of the nanoparticles. At $3 \mathrm{~g} / \mathrm{L}$ PVP, the cell viability increased greatly to levels higher than for pristine HA nanoparticles. These findings clearly indicate that the morphology, particle size, and uniformity greatly influence the functional properties of the nanoparticle. High aspect ratio nanoparticles have been reported to have selective surface chemistry that enhances the functional activity of these materials. A high aspect ratio of HA nanoparticles has been shown to improve the mechanical strength while improving dental adhesiveness $[19,20]$. Additionally, the elongated structure caused by the high aspect ratio has often been shown to lead to specific intrinsic properties that can be effectively used for interactions with cells and biomolecules in new ways [1]. The in vitro cellular analysis in this study clearly demonstrated that uniformity and high aspect ratio played a role in bioactivity. The high aspect ratio provides a greater surface area due to its narrow structure and may, therefore, lead to better adhesion of cells in these structures. Moreover, the uniformity of the structure enhances the cell viability as confirmed by our comparison of 2 and $3 \mathrm{~g} / \mathrm{L}$ PVP added samples with pristine and $4 \mathrm{~g} / \mathrm{L}$ PVP added samples. As shown in Figure 8 the density of cell growth depended on the uniformity of the samples. Higher aspect ratio nanoparticles with uniform size distribution having a large surface area available for interaction would cause higher cell viability than nonuniformly distributed particles. Since the size distribution of the 2 and $3 \mathrm{~g} / \mathrm{L}$ PVP added samples varied greatly, the cell viability decreased in these samples. Hence, we found that controlling the uniformity and aspect ratio enables the bioactivity of a biomaterial to be tuned as desired. 


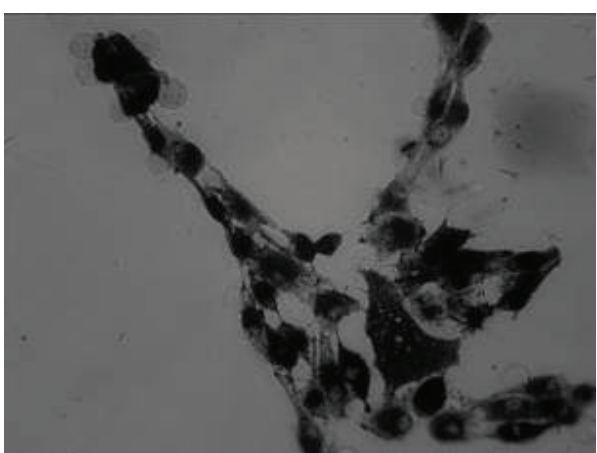

(a)

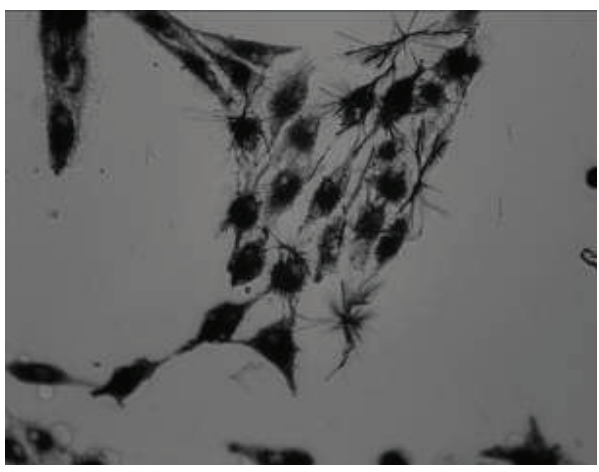

(c)

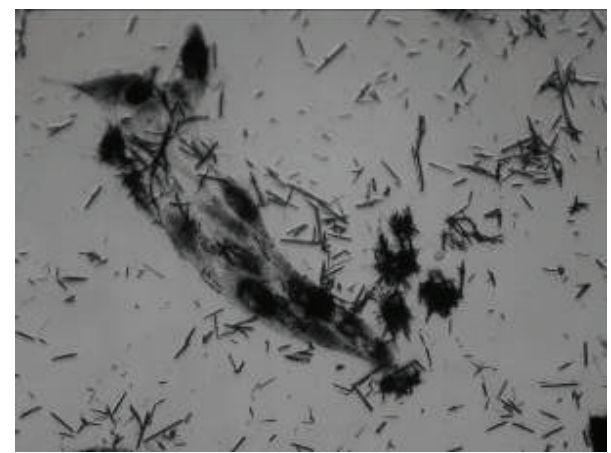

(b)

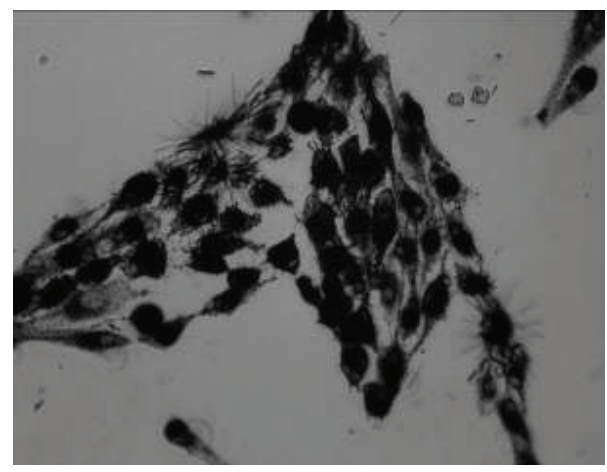

(d)

FIGURE 8: Optical micrograph of MG-63 cell spreading on the high aspect ratio HA nanorods: (a) pristine and (b) 2, (c) 3, and (d) 4 g/L PVP addition.

\section{Summary}

We successfully synthesized high aspect ratio HA nanorods in the presence of polyvinylpyrrolidone (PVP). There was a clear increase in the length of the nanorods with the addition of PVP that was plainly visible in the morphological analysis. The intensity of the (002) plane also increased considerably suggesting that growth occurred in the $c$-axis direction. The aspect ratio of the nanorods was very high relative to the pristine HA nanorods. Accordingly, this type of material with tunable morphology will have useful biomedical applications.

\section{Conflict of Interests}

The authors have no conflict of interests to declare.

\section{Acknowledgment}

This research was supported by the Global Special Technology Development (Grant no. 10044611) of Ministry of Trade, Industry \& Energy in 2013-2015.

\section{References}

[1] L. A. Bauer, N. S. Birenbaum, and G. J. Meyer, "Biological applications of high aspect ratio nanoparticles," Journal of Materials Chemistry, vol. 14, no. 4, pp. 517-526, 2004.
[2] M. Cargnello, T. R. Gordon, and C. B. Murray, "Solution-phase synthesis of titanium dioxide nanoparticles and nanocrystals," Chemical Reviews, vol. 114, no. 19, pp. 9319-9345, 2014.

[3] Z. Niu and Y. Li, "Removal and utilization of capping agents in nanocatalysis," Chemistry of Materials, vol. 26, no. 1, pp. 72-83, 2014.

[4] A. J. Nathanael, D. Mangalaraj, P. C. Chen, and N. Ponpandian, "Enhanced mechanical strength of hydroxyapatite nanorods reinforced with polyethylene," Journal of Nanoparticle Research, vol. 13, no. 5, pp. 1841-1853, 2011.

[5] A. J. Nathanael, S. I. Hong, D. Mangalaraj, N. Ponpandian, and P. C. Chen, "Template-free growth of novel hydroxyapatite nanorings: formation mechanism and their enhanced functional properties," Crystal Growth and Design, vol. 12, no. 7, pp. 3565-3574, 2012.

[6] J. Huang, Y. W. Lin, X. W. Fu et al., "Development of nano-sized hydroxyapatite reinforced composites for tissue engineering scaffolds," Journal of Materials Science: Materials in Medicine, vol. 18, no. 11, pp. 2151-2157, 2007.

[7] K.-U. Lewandrowski, S. P. Bondre, D. L. Wise, and D. J. Trantolo, "Enhanced bioactivity of a poly(propylene fumarate) bone graft substitute by augmentation with nano-hydroxyapatite," BioMedical Materials and Engineering, vol. 13, no. 2, pp. 115-124, 2003.

[8] A. J. Nathanael, S. I. Hong, D. Mangalaraj, and P. C. Chen, "Large scale synthesis of hydroxyapatite nanospheres by high gravity method," Chemical Engineering Journal, vol. 173, no. 3, pp. 846-854, 2011. 
[9] W. Qingqing, Z. Gaoling, and H. Gaorong, "Synthesis of single crystalline CdS nanorods by a PVP-assisted solvothermal method," Materials Letters, vol. 59, no. 21, pp. 2625-2629, 2005.

[10] A. J. Nathanael, S. S. Han, and T. H. Oh, "Polymer-assisted hydrothermal synthesis of hierarchically arranged hydroxyapatite nanoceramic," Journal of Nanomaterials, vol. 2013, Article ID 962026, 8 pages, 2013.

[11] J. Zhang, H. Liu, Z. Wang, N. Ming, Z. Li, and A. S. Biris, "Polyvinylpyrrolidone-directed crystallization of $\mathrm{ZnO}$ with tunable morphology and band gap," Advanced Functional Materials, vol. 17, no. 18, pp. 3897-3905, 2007.

[12] S. H. Jeon, P. Xu, B. Zhang et al., "Polymer-assisted preparation of metal nanoparticles with controlled size and morphology," Journal of Materials Chemistry, vol. 21, no. 8, pp. 2550-2554, 2011.

[13] Y. Sun, B. Mayers, T. Herricks, and Y. Xia, "Polyol synthesis of uniform silver nanowires: a plausible growth mechanism and the supporting evidence," Nano Letters, vol. 3, no. 7, pp. 955960, 2003.

[14] A. J. Nathanael, D. Mangalaraj, S. I. Hong, Y. Masuda, Y. H. Rhee, and H. W. Kim, "Influence of fluorine substitution on the morphology and structure of hydroxyapatite nanocrystals prepared by hydrothermal method," Materials Chemistry and Physics, vol. 137, no. 3, pp. 967-976, 2013.

[15] G. Xu, X. L. Wang, and G. Z. Liu, "Facile solvothermal synthesis of abnormal growth of one-dimensional $\mathrm{ZnO}$ nanostructures by ring-opening reaction of polyvinylpyrrolidone," Applied Surface Science, vol. 329, pp. 137-142, 2015.

[16] Y. K. Du, P. Yang, Z. G. Mou, N. P. Hua, and L. Jiang, "Thermal decomposition behaviors of PVP coated on platinum nanoparticles," Journal of Applied Polymer Science, vol. 99, no. 1, pp. 23-26, 2006.

[17] T. Gutul, E. Rusu, N. Condur, V. Ursaki, E. Goncearenco, and P. Vlazan, "Preparation of poly(N-vinylpyrrolidone)-stabilized zno colloid nanoparticles," Beilstein Journal of Nanotechnology, vol. 5, no. 1, pp. 402-406, 2014.

[18] Y. Sun, B. Gates, B. Mayers, and Y. Xia, "Crystalline silver nanowires by soft solution processing," Nano Letters, vol. 2, no. 2, pp. 165-168, 2002.

[19] M. Sadat-Shojai, M. Atai, A. Nodehi, and L. N. Khanlar, "Hydroxyapatite nanorods as novel fillers for improving the properties of dental adhesives: synthesis and application," Dental Materials, vol. 26, no. 5, pp. 471-482, 2010.

[20] L. Chen, Q. Yu, Y. Wang, and H. Li, "BisGMA/TEGDMA dental composite containing high aspect-ratio hydroxyapatite nanofibers," Dental Materials, vol. 27, no. 11, pp. 1187-1195, 2011. 

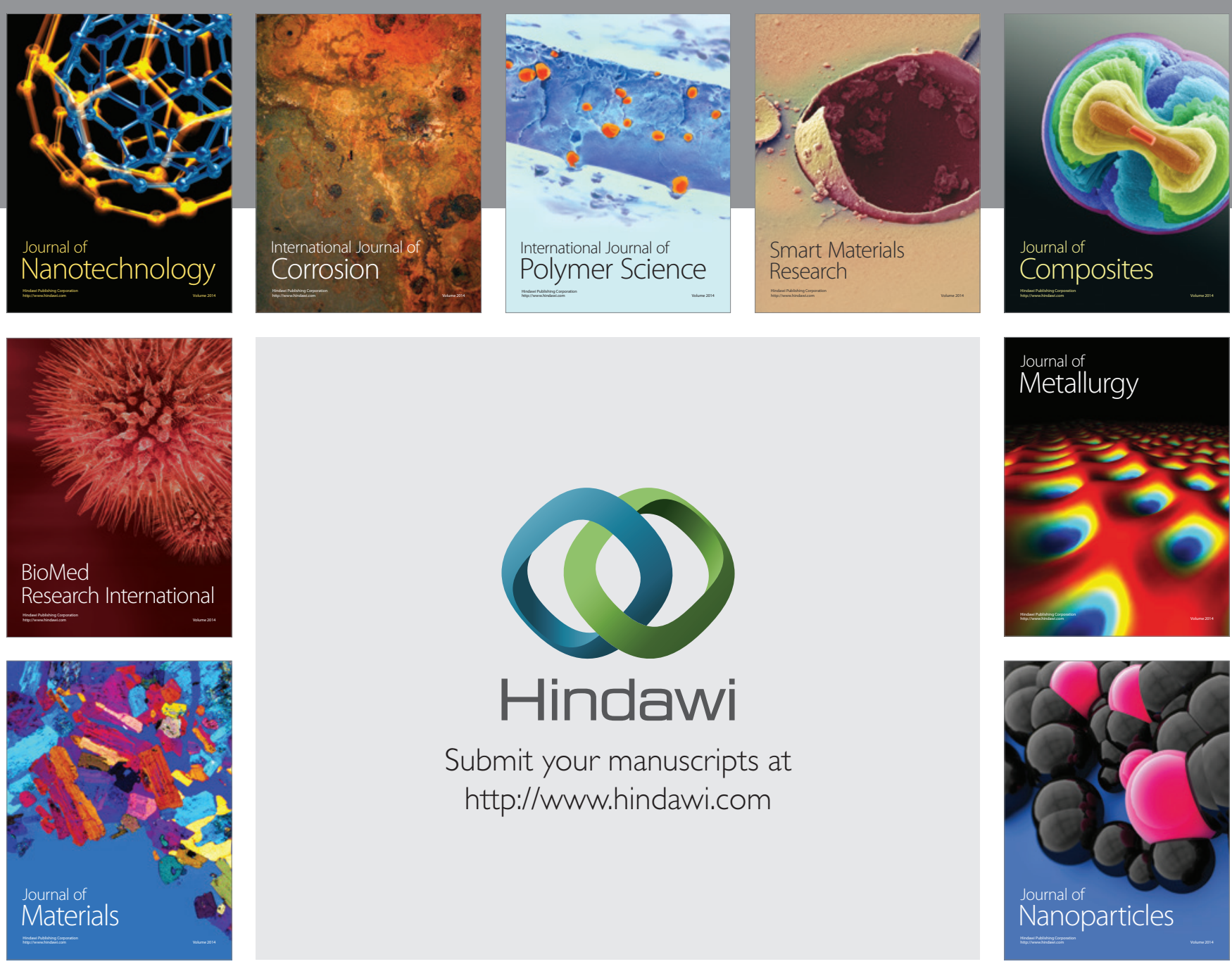

Submit your manuscripts at http://www.hindawi.com
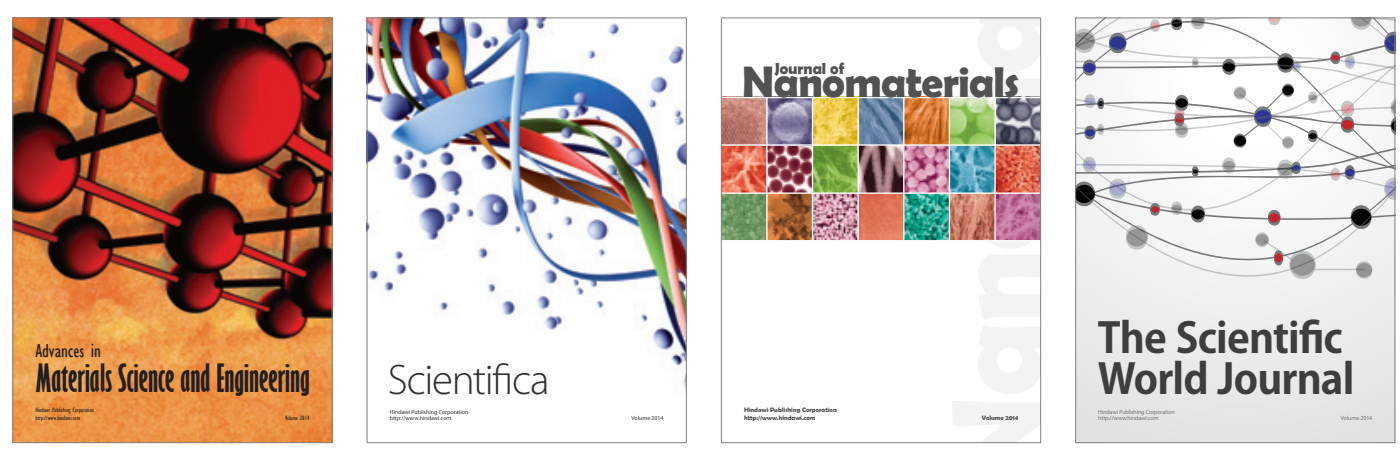

\section{The Scientific World Journal}
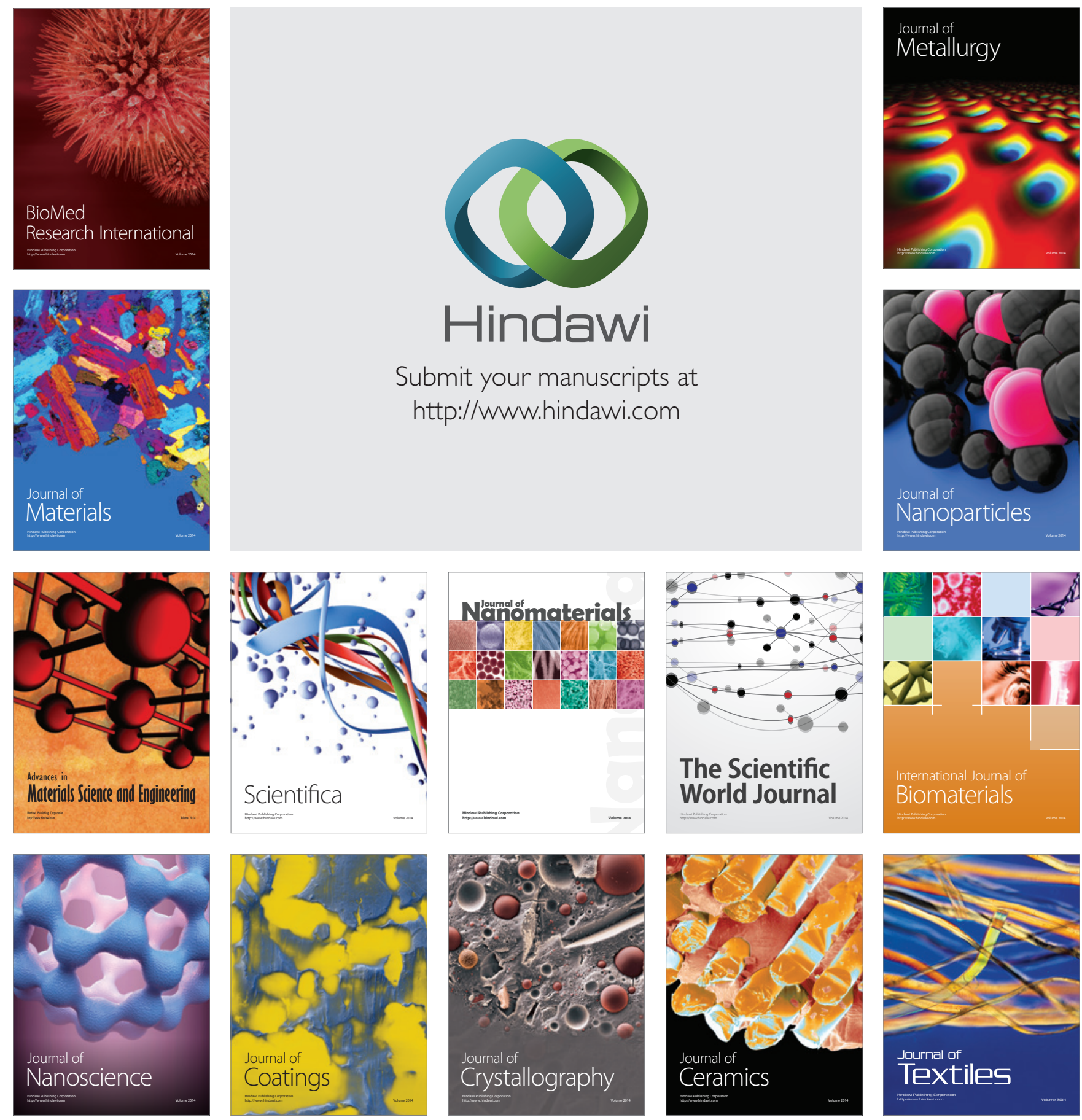